\title{
Los índices de medición de la Reputación Corporativa en la cadena de valor de las empresas de comunicación, una propuesta
}

\author{
Jaime Alberto Orozco-Toro \\ Universidad Pontificia Bolivariana. Medellín, Colombia. Facultad de Publicidad. \\ jaime.orozco@upb.edu.co
}

\begin{abstract}
Carme Ferré-Pavia
Universidad Autónoma de Barcelona. Barcelona-España. Departamento de Medios, Comunicación y Cultura. carme.ferre@uab.cat
\end{abstract}

Recibido: 17 de julio de 2017. Aceptado: 18 de octubre de 2017.

\section{Resumen}

La necesidad de conocer la valoración que hacen los grupos de interés de las empresas aumenta en contextos cada vez más competitivos, lo que hace que evaluar la Reputación Corporativa (RC) sea una tarea primordial en el ámbito de la comunicación corporativa. A pesar de la existencia de variados índices de medición de la RC, ninguno de ellos responde a la evaluación de toda la cadena de valor, ya que en su mayoría toman como muestra a tan solo algunos de los stakeholders. Adicionalmente, no están difundidos índices de Reputación sectoriales o enfocados a un sector económico concreto.

Este artículo es una propuesta metodológica que presenta un Índice de Reputación Corporativa para Empresas de Comunicación (denominado IRCEC) construido a partir del análisis de otros índices académicos y empresariales existentes. De esta forma, el desarrollo metodológico ha permitido el estudio de diferentes índices de medición en el ámbito teórico y empresarial, los cuales utilizan dimensiones y atributos para la implementación de sus herramientas de evaluación, como se hace usualmente en todos los índices. Los resultados de la investigación muestran la propuesta del IRCEC detallada por esas dimensiones y atributos. Se concluye que cada una de las partes interesadas puede tener una valoración diferenciada de la RC. La evaluación segmentada por stakeholders aparece como la vía más recomendable para posteriormente comparar percepciones y emprender estrategias diferenciadas para cada grupo de interés. Una estrategia encaminada a mejorar la RC de una empresa deberá identificar los valores intangibles de la marca que son importantes para sus grupos de interés.

Palabras clave: cadena de valor, empresas de comunicación, índices de reputación, reputación corporativa, stakeholders. 


\title{
Measuring corporate reputation along the value chain of communication companies: a proposal
}

\begin{abstract}
In an increasingly competitive context, the need to learn how interest groups assess companies is on the rise. This means that evaluating corporate reputation (CR) is a crucial task for corporate communications. Although there exist various indexes for measuring corporate reputation, none consider the entirety of the value chain, since most sample only a few stakeholders. Additionally, indexes gauging sector reputation or focusing on concrete economic fields are not widely available. This article is a methodological proposal that presents an Index of Corporate Reputation for Communication Companies (ICRCC), constructed after an analysis of existing academic and company indexes. Our methodological approach allowed us to study, in theoretical and corporate contexts, different measurement indexes that employ dimensions and indicators in implementing their evaluation tools, as indexes usually do. The results of our investigation show our ICRCC proposal broken down into these dimensions and indicators. We conclude that each interested party can have a distinct view of corporate reputation. Segmenting evaluations by stakeholders appears to be the most advisable way to then compare perceptions and elaborate differentiated strategies for each interest group. A strategy that means to improve a company's corporate reputation must identify the intangible brand values that interest groups care about.
\end{abstract}

Keywords: value chain, communication companies, reputation indexes, corporate reputation, stakeholders.

\section{Os índices de medição da Reputação Corporativa na cadeia de valor das empresas de comunicação, uma proposta}

\section{Resumo}

A necessidade de conhecer a valoração que fazem os grupos de interesse das empresas aumenta em contextos cada vez mais competitivos, o que faz com que avaliar a Reputação Corporativa (RC) seja uma tarefa primordial no âmbito da comunicação corporativa. Apesar da existência de variados índices de medição da RC, nenhum deles e responde a avaliação de toda a cadeia de valor, já que em sua maioria tomam como mostra apenas a alguns dos stakeholders. Adicionalmente, não estão difundidos índices de Reputação setoriais ou focados a um setor econômico concreto.

Este artigo é uma proposta metodológica que apresenta um Índice de Reputação Corporativa para Empresas de Comunicação (denominado IRCEC) construído a partir da análise de outros índices acadêmicos e empresariais existentes. Dessa forma, o desenvolvimento metodológico tem permitido o estudo de diferentes índices de medição no âmbito teórico e empresarial, os quais utilizam dimensões e atributos para a implementação de suas ferramentas de avaliação, como se faz usualmente em todos os índices. Os resultados da pesquisa mostram a proposta do IRCEC detalhada por essas dimensões e atributos. Conclui-se que cada uma das partes interessadas pode ter uma valoração diferenciada da RC. A avaliação segmentada por stakeholders aparece como a via mais recomendável para, posteriormente, comparar percepções e empreender estratégias diferenciadas para cada grupo de interesse. Uma estratégia destinada a melhorar a RC de uma empresa deverá identificar os valores intangíveis da marca que são importantes para seus grupos de interesse.

Palavras chave: cadeia de valor, empresas de comunicação, índices de reputação, reputação corporativa, stakeholders. 


\section{Introducción}

La Reputación Corporativa (RC) no es un concepto nuevo para la empresa, si bien es cierto que los mayores avances en materia de investigación y desarrollo de su análisis se han generado en los últimos años. Así lo corrobora Larkin (2003) al destacar"the academic research is still in its infancy"' (p. 40).

Aunque es posible que anteriormente no se diera a la $\mathrm{RC}$ el valor que tiene, hoy en día los intangibles, activos que producen beneficios a la empresa, adquieren una gran importancia debido a que "la marca es la visible punta del iceberg [...] pero la reputación permanece escondida al acecho bajo la superficie" (Hannington, 2006, p. 37). Y no solo cobra importancia la RC por haberse convertido en un valor fundamental en la gestión de los intangibles (Tejedo-Romero \& Ferraz, 2015), sino por los vínculos que tiene con la identidad, la imagen, la marca, e inclusive por ser considerada como "the missing link between business and ethics"2 (Jackson, 2004, p. 86). Además, como aclara Alloza (2015), de estos intangibles "depende el éxito para la construcción de proyectos sostenibles a largo plazo" (p. 171).

Algunos de los autores más importantes relacionados con la RC (Sanz de la Tajada, 1994; Fombrun, 1996; Van Riel, 1997; Schultz, Hatch \& Larsen, 2000; Dowling, 2001; Quevedo, 2003; Villafañe, 2004; Freeman, 2006; Hannington, 2006; Álvarez, 2008; Firestein, 2009; Doorley \& García, 2011; Komisarjevsky, 2012), coinciden en que los aspectos más importantes de la RC son: percepciones, valores, atributos, comportamiento, intangibles y stakeholders, entre otros. También, en los últimos años, se han desarrollado corrientes con enfoque en la medición de la RC (Argenti, 2014; Huang, Wang, Hsu, Zhang \& Yang, 2015; Valenzuela, Jara-Bertin \& Villegas, 2015; Paranaíba, Souza, Marques \& Miranda, 2016).

Esta investigación propone un modelo de análisis de la reputación corporativa adaptable a las empresas de comunicación. La comparativa con otros modelos y la adaptación a la completa cadena de valor, lo que significa atender a todos sus stakeholders, marcan dos de sus valores destacables. Los resultados, en un recorrido metodológico, pasan por las dimensiones y atributos seleccionados, y a partir de su justificación se añaden las preguntas que confeccionarían un cuestionario adaptable al modelo.

Las empresas de comunicación, a las que se ha tardado mucho en reclamar una implementación de su RSC (Orozco-Toro \& Ferré-Pavia, 2017), han sido y son afectadas por crisis muy duras, de modelo de negocio, consumo y también de reputación (Ingenhoff \& Koelling, 2012; Delgado \& Olarte, 2012; Fernández, 2013). En este contexto, un modelo de análisis adaptado al sector puede ser de ayuda para contar con datos cercanos a los efectos de la reputación en toda su cadena de valor.

\footnotetext{
"La investigación académica continúa en la infancia" (trad. de los autores).

2 "El eslabón perdido entre los negocios y la ética" (trad. de los autores).
} 


\section{Marco teórico}

Tratándose de una propuesta para medir la RC en toda la cadena de valor de una empresa, este marco teórico se centra en el vínculo entre los stakeholders y la reputación corporativa que otorgan a las empresas, tanto desde el punto de vista de la gestión de los intangibles de esta reputación, de los modelos que hasta ahora la han evaluado, como del foco concreto aplicado a las empresas de comunicación.

\section{La gestión de intangibles}

El vínculo RC-stakeholders es aquello a lo que más atención se le presta en las investigaciones, debido al valor preponderante que los grupos de interés generan en organizaciones de cualquier índole. Pero sobre todo porque son sus percepciones, sus actuaciones y sus decisiones las que modifican la RC, bien sea de una empresa, de una marca, de un producto o de un servicio.

El vínculo entre los stakeholders y la RC es tan fuerte que el mismo Costa (2009) precisa que la reivindicación de la $\mathrm{RC}$ en los últimos años ha sido originada por la irrupción de la noción de grupos de interés. Aunque Caruana (2008) revele que la $\mathrm{RC}$ es el resultado de las percepciones de todos los stakeholders, se debe considerar oportuno investigar y definir cuáles son las percepciones de cada uno de los grupos de interés de la compañía. Según sea el caso, una misma marca puede tener diferentes reputaciones dependiendo de las percepciones de sus grupos de interés. Esta es la razón por la cual se considera la RC como un valor difícil de controlar, debido a que "it is the publics that build reputation, not organizations themselves"3 (Aula \& Mantere, 2008, p. 210).

Esas varias reputaciones se construyen desde el reconocimiento de los valores intangibles hasta la estrategia corporativa que se implemente. Los principales escenarios en los que se crea reputación comienzan desde la generación de objetivos, una identidad bien establecida, una estrategia idónea, uso de los medios de comunicación adecuados, comunicación transparente, estrecha relación con los stakeholders, entre muchos otros factores.

Esta gestión de intangibles va más allá de la materialidad de los productos y se adentra en el plano de la satisfacción de necesidades de los grupos de interés. En este caso, los intangibles deberán tener un carácter estratégico con el fin de diferenciarse de los que utilizan los competidores (Rodríguez \& Alfaro, 2004). Esta puede ser una primera pregunta de las empresas cuando intentan construir RC: cuáles son los valores intangibles que pueden generar diferencia con la competencia. Posteriormente, es vital pensar en los grupos de interés (Gaultier, Louisot \& Rayner, 2009), pues para ellos

\footnotetext{
3 “Son sus públicos, y no ella misma, quienes construyen la reputación de una empresa" (trad. de los autores).
} 
"an organisation enjoys a good reputation when it consistently meets or exceeds the expectations of its stakeholders" 4 (p. 162).

El proceso pasa por otros escenarios de igual relevancia, como es el caso de la implementación estratégica. Tanto para Gable (2008) como para Firestein (2009), una fuerte reputación requiere basarse en una estrategia a largo plazo y en acciones tácticas que permitan movimientos rápidos ante los cambios del mercado. Bajo estas condiciones, una amplia variedad de empresas ha demostrado su interés en medir el resultado de sus estrategias, no solamente desde el punto de vista bursátil, sino desde perspectivas complementarias como la imagen o la reputación. Así, son muchos investigadores que han abogado por la importancia de evaluar la reputación de las empresas (Nomen, 2005; Hannington, 2006; Salinas, 2007; Lindemann, 2010; Doorley \& Garcia, 2011).

\section{Medir la RC}

Evaluar la RC se ha convertido en una gestión indispensable para organizaciones e investigadores. Esta necesidad proviene de la idea que señala Lizcano (2006), de que "los recursos y actividades intangibles han de poder ser representados en una serie de indicadores que hagan posible su medición y comparabilidad" (p. 165). La valoración de la RC ha sido un tema recurrente a partir de la década de 1990 debido a la evidencia de que los intangibles tienen un valor financiero (Olins, 2000) y que una buena reputación es la base para hacer frente a la creciente competitividad de los mercados globales (Fombrun \& Rindova, 2000; Sarstedt, Wilczynski, \& Melewar, 2013). Adicionalmente hay quienes consideran que el mayor interés acerca de la RC es su posibilidad de medición, razón por la cual se ha convertido en una oportunidad para la generación de nuevos índices de medición (Sanna, 2013).

En España, el abanderado de la investigación relacionada con la RC ha sido Villafañe (2004), para quien "la reputación corporativa no solo se puede evaluar y es mesurable, sino que también se puede verificar a través de hechos sólidos que permiten su contraste con los de otras organizaciones" (p. 32). Pero lo que hace este investigador para valorar la reputación de una empresa se suscribe solamente a entrevistas a altos directivos de empresas sin tener en cuenta otros grupos de interés.

Desde otra perspectiva, Larkin (2003) asume que los intangibles de una compañía se pueden evaluar tomando como base la diferencia entre su valor del mercado y su valor en los libros contables. Es posible que lo que este autor esté cuantificando no

4 "Una empresa goza de una buena reputación cuando cumple o supera de manera consistente las expectativas de sus grupos de interés" (trad. de los autores). 
sean los valores intangibles a través de la RC, sino el goodwill ${ }^{5}$, pues se trata de un valor asignado al realizar una transacción por una empresa.

Por su parte, Nomen (2005) basa su propuesta para evaluar los intangibles en la siguiente aserción: "El valor económico de una cosa es una percepción del observador que corresponde a la capacidad que otorga a la cosa para ejecutar funciones, percibidas con capacidad de satisfacer necesidades" (p. 30). Siendo una definición poco ortodoxa, plantea dos acepciones importantes: la idea de percepciones y capacidad otorgada. Es decir, para medir un intangible se deben valorar las percepciones subjetivas de los stakeholders de acuerdo con las necesidades que dicho intangible satisfaga. La teoría de Nomen no es aceptada por Lindemann (2010), ya que considera que estos modelos no atribuyen un valor financiero, sino que terminan midiendo el comportamiento y la actitud de los consumidores.

Las posiciones más significativas de investigadores proponen la utilización de métodos cualitativos y cuantitativos para evaluar la reputación. Una de las propuestas se encamina a la combinación de diferentes modelos de otros investigadores para luego preguntar a los grupos de interés por sus percepciones directamente sobre la reputación (Earl \& Waddington, 2012), entretanto Hannington (2006) es quien mejor desarrolla una propuesta de evaluación de los intangibles con el fin de conocer la reputación de una compañía. Para este investigador, se deben cumplir unas circunstancias básicas. La primera es evaluar a todos los stakeholders. Esta recomendación es muy importante, pues muchos de los métodos que existen en la actualidad para medir la reputación de las empresas se basan en las percepciones de un solo grupo de interés (Ruiz, Esteban \& Gutiérrez, 2014). Incluso hay investigadores que se preguntan "si los diferentes grupos de stakeholders perciben la reputación de igual manera o si, por el contrario, existen diferentes clases de reputación" (Sanna, 2013, p. 97). En este sentido, Carrió (2013) considera que los métodos multistakeholder "aportan una información integral del estado de la reputación de una organización permitiendo alinear de manera efectiva los parámetros de la reputación a nivel de cada público particular" (p. 101). Pérez \& Rodríguez del Bosque (2014) aclaran, en una revisión de la teoría al respecto, que mientras en el ámbito de la organización de las empresas se "identifica una cierta tendencia de los investigadores a definir la identidad, imagen y reputación en función de los grupos de interés [...], por el contrario, en los ámbitos de la comunicación corporativa y del marketing no se realiza dicha distinción de grupos de interés, definiéndose los conceptos de manera genérica" (p. 99). La propuesta de contar con todos ellos y ponderar elementos cualitativos sienta las bases para construir una herramienta que permita evaluar la RC.

Desde la perspectiva de dichas herramientas de medición de la RC, se cuenta con diferentes modelos de evaluación (Raithel \& Schwaiger, 2015). Los modelos vincula-

Se considera tradicionalmente como el buen nombre de la empresa, aunque el concepto está más dirigido a los estados contables de la compañía y su relación en el momento de su venta. 
dos con la comunicación se han desarrollado desde dos perspectivas: una académica y otra desde la consultoría; ambas buscan mejorar la gestión empresarial (Garrido, 2004). Debido a la dificultad que conlleva esta actividad, los medios de comunicación han optado por realizar únicamente mediciones de audiencia. A pesar de las dificultades propias de la creación de herramientas que determinen los efectos de la comunicación de las organizaciones, no se pueden escatimar esfuerzos en la búsqueda de instrumentos de evaluación. Estos modelos permitirían cumplir con una doble función: una dimensión diagnóstica y otra de plan correctivo o recomendaciones (Etkin, 2008). Ahí su importancia, ya que no solo permite una radiografía de las percepciones, sino que se convierte en el primer paso de un plan estratégico.

No son pocos los que consideran que la medición de la RC, por tratarse de un intangible, genera grandes dificultades. Esto se basa en la idea de que la RC no es una ciencia y que no se puede asignar un valor matemático a las emociones de los stakeholders (Griffin, 2009). Por su parte, Davies Chun, Vinhas \& Roper (2003) aseguran que la RC puede medirse, pero proponen nuevos parámetros para su ejecución: “a tenet of the reputation paradigm is that reputation, however ethereal, can be measured. But there are problems with existing measures" 6 (p. 72). Aunque estos investigadores no realizan una propuesta de modelo, consideran que lo más importante es que las nuevas herramientas "should be capable of use with as many different categories of stakeholders as possible"7 (p. 140).

La idea de que la RC no se puede medir teniendo como base un solo grupo de interés es la mayor crítica que se hace a los modelos existentes. En esto radica la necesidad de implicar a toda la cadena de valor en la evaluación de la RC. Crear un modelo de evaluación de la RC no solo permitiría a las compañías conocer las percepciones de todos los grupos de interés, sino que se convertiría en "una pieza clave para crear un sistema de gestión reputacional que permita mejorar el comportamiento de todas las áreas de la empresa” (Hernández, Losada \& Macías, 2007, p. 113) y la creación de un "sistema de gestión reputacional que persiga una mejora progresiva de la percepción de los diversos stakeholders de una organización basada en su capacidad de responder a las expectativas" (Alloza, 2006, p. 183).

En este sentido de la medición de la RC en diferentes tipos de stakeholders, Argenti (2014) asume que para evaluar la reputación de una organización deben analizarse las percepciones de todos sus grupos de interés, y es enfático al aclarar que "aunque no a todo el mundo le vale la misma talla, cuando se trata de medir, todas las organizaciones necesitan este tipo de estudios sobre los grupos de interés con los que se relacionan" (p.211).

Los posibles beneficios de una evaluación de la RC que sugieren Hernández et al. (2007) y Alloza (2006) posibilitarían el propio desempeño, por ejemplo, de la

\footnotetext{
6 "Uno de los principios del paradigma de la reputación, de todos modos etérea, es que puede ser medida. Pero existen problemas con las mediciones" (trad. de los autores).

7 "Sean capaces de aplicarse con cuántas categorías diferentes de stakeholders sea posible" (trad. de los autores).
} 
Responsabilidad Social Corporativa, ya que es una forma fundamental para conocer las expectativas y necesidades de los stakeholders.

\section{Modelos de evaluación de la RC}

En el ámbito empresarial existen diversos modelos de evaluación de la RC. En el mundo, los modelos más reconocidos son el WMAC de la Revista Fortune, el Reputation Quotient de Harris Interactive y el RepTrak del Reputation Institute. En España y algunos países de América Latina también se utiliza el Merco de Villafañe y Asociados. Estos cuatro modelos basan sus estudios en la utilización de dimensiones y atributos. A manera de información comparativa, se desglosan las dimensiones de cada uno (tabla 1).

Tabla 1. Dimensiones de los modelos de evaluación de la R ${ }^{8}$.

\begin{tabular}{ll}
\hline Modelo & Dimensiones \\
\hline & Productos y servicios \\
& Visión y liderazgo \\
& Entorno de Trabajo \\
& Responsabilidad Social y Medioambiental \\
& Rendimiento Financiero \\
\hline & Calidad Productos y Servicios \\
& Reputación interna \\
& Ética y Responsabilidad Social Corporativa \\
& Rendimiento Económico-Financiero \\
& Innovación \\
& Dimensión global y presencia internacional \\
\hline & Productos y Servicios \\
& Visión y Liderazgo \\
& Entorno de Trabajo \\
& Responsabilidad Social y Medioambiental \\
& Rendimiento Financiero \\
& Atractivo Emocional \\
\hline RQ & Oferta \\
& Liderazgo \\
& Trabajo \\
& Gobierno \\
& Ciudadanía \\
& Innovación \\
& Finanzas \\
\hline
\end{tabular}

*Los índices profesionales son anuales, por lo que no se indicará el año cuando aparezcan.

El World's Most Admired Companies (WMAC) ha sido uno de los más criticados. Los investigadores aducen que no se basa en fundamentos teóricos, que no es una herramienta que permita mejorar la RC de las empresas y que no evalúa toda la cadena de valor (Davies et al., 2003). En la misma vía, Doorley \& García (2011) reiteran que el WMAC "surveys only three constituencies: senior executives, (outside) board

\footnotetext{
8 Todas las tablas son de elaboración propia.
} 
members, and securities analysts. A more comprehensive approach would include surveying all the major constituencies, including employees, customers, and the press" (p. 8). El Monitor Empresarial de Reputación Corporativa (Merco) tiene el mismo problema, debido a que solo toma las opiniones de directivos y de expertos sin tener presentes a otros grupos de interés (Merino \& Pintado, 2009; Fernández, 2011).

En relación con el Reputation Quotient (RQ), los detractores asumen que el modelo utiliza exclusivamente criterios cualitativos con una medida simple de atributos (Alloza, 2006). En la misma línea, Caruana (2008) cuestiona que "the operationalization of the RQ instrument is inadequately grounded in theory and it is hard to see how the definition used has guided instrument development"10 (p. 203).

Bajo la opinión de los expertos, el modelo Reptrak es el que menos inconvenientes tiene, entre otras razones porque se trata de un modelo cuantitativo que permite calcular la importancia de los atributos y las dimensiones (Hernández et al., 2007). Los grupos de interés a los que se aplica el modelo son básicamente directivos a través de entrevistas en profundidad y a público en general a través de focus groups (López \& Sebastián, 2009).

Una conclusión que se puede sacar del análisis general de estos métodos de evaluación de la RC es que no hay modelos infalibles. La valoración de los intangibles de una empresa siempre será una tarea dispendiosa y de alguna manera subjetiva. Por eso es necesario continuar la búsqueda de metodologías que permitan evaluar correctamente la RC.

\section{RC de empresas de comunicación y sus stakeholders}

Existe una cierta desatención por parte de investigadores respecto a los aspectos relacionados con la RSC de las empresas de comunicación. Situación similar se vive en la RC, pues así lo demuestran Arrese \& Baigorri (2011) cuando expresan "in conclusion, there has been little interest amongst Spanish academics in analyzing the links between corporate reputation and media coverage"11 (p. 171). Sin embargo, en los últimos tiempos se viene prestando un poco más de interés al tema de la RC:

Las empresas públicas televisivas de radiodifusión vienen realizando desde mediados de los noventa verdaderos esfuerzos por construir fuertes reputaciones de marca, sobre una plataforma de posicionamiento basado en el valor y en la calidad. En este sentido, en el caso de las cadenas públicas, existe una creciente preocupación por mostrarse como instituciones educativas y culturales de alta reputación (Vila \& Küster, 2013, p. 337).

\footnotetext{
9 "Encuesta solo a tres grupos: directivos senior, miembros del consejo (externos) y analistas de seguridad. Un enfoque más amplio incluiría a los grupos más importantes, empleados, clientes y medios incluidos" (trad. de los autores).

10 "La puesta en funcionamiento de la herramienta RQ no está debidamente fundamentada y es difícil ver como la definición utilizada ha guiado el desarrollo de instrumentos" (trad. de los autores).

11 "En conclusión, ha existido poco interés en los académicos españoles en analizar el enlace entre la reputación corporativa y la cobertura de los medios" (trad. de los autores).
} 
Esta gestión de la RC en las empresas de comunicación implicaría estructurar las propias compañías desde su estrategia corporativa, e incluso supondría modificaciones en su estructura organizacional. Aunque se pueden encontrar algunos casos en los que se realizan cambios significativos en los medios de comunicación, muchos de ellos obedecen más a necesidades económicas que a la búsqueda de mejoras en su gestión de RC o incluso de RSC.

Estas condiciones sobre la gestión de la RC en empresas de comunicación están mediadas en especial por la irrupción de la teoría de los stakeholders. Basados en estas nuevas condiciones a las que se enfrenta la empresa, se puede reconocer que sea la RC uno de los factores que más han sido afectados por el nuevo poder que tienen los stakeholders. Esta idea se puede corroborar con la afirmación de Aguilera \& Baños (2016), para quienes el mayor reto de las empresas hoy en día es el público al que se dirigen:

Un público compuesto por personas más interesadas en informarse, que pueden acceder
miles de mensajes sobre una marca que no ha emitido el anunciante sino otros usuarios,
que cada vez creen menos en la publicidad convencional, que pueden convertir una expe-
riencia de uso en el mejor o en el peor mensaje sobre el producto, que prefieren llegar a los
mensajes que les interesan en lugar de verse forzados a recibir contenidos que no desean,
que quieren ser escuchados por las marcas (p. 17).

Finalmente, es necesario advertir que la aparición de la teoría de los stakeholders no ha dejado de lado la importancia de los usuarios de las empresas de comunicación. Dentro de estas circunstancias, Aguilera \& Baños (2016) reconocen que se deben hacer esfuerzos para lograr una relación duradera con los grupos de interés, entre los cuales se encuentra el engagement con los usuarios:

Pocas funciones empresariales han sufrido una revolución tan profunda como la del marketing, cuyo objetivo es ahora el de generar engagement para estrechar los vínculos afectivos, cognitivos y de comportamiento entre la marca y el consumidor. Y cada tipo de marketing conlleva su propio tipo de comunicaciones. Comunicaciones relevantes para los consumidores sustentadas, frecuentemente, en el valor añadido que aportan a los públicos (p. 26).

No es de extrañar que cada día las empresas estén más atentas a los diferentes grupos de interés que las rodean, y aunque aún se encuentra un precario desarrollo de las teorías vinculadas con los stakeholders, tendrán que mejorarse las concepciones académicas sobre el tema, pero sobre todo las acciones que generen una relación ética de mutuo beneficio entre la organización y sus diferentes partes interesadas. Esto es, si cabe, más importante en empresas en crisis global por el modelo de cambio de negocio del mundo comunicativo, las audiencias fragmentadas, la competencia de las redes sociales y las crisis éticas de algunos medios de comunicación (Orozco-Toro \& Ferré-Pavia, 2012). 


\section{Materiales y método}

El objetivo de esta investigación es ofrecer una herramienta que mejore los procesos de evaluación de la RC en empresas de comunicación. Para generar la propuesta del IRCEC (Índice de Reputación Corporativa para Empresas de Comunicación) se partió del análisis de otros modelos de evaluación que se implementan en los mundos empresarial y académico.

Para ello, se analizó un total de 15 índices de RC, 11 en el ámbito académico y 4 empresariales. Los índices empresariales se han seleccionado por su difusión en los medios de comunicación y por ser los más utilizados por compañías, pues se emiten rankings para evidenciar la RC de las empresas alrededor del mundo. Los índices académicos se han seleccionado entre los que han realizado alguna propuesta de evaluación de la RC, independientemente de los grupos de interés a los que evalúan.

Usualmente, los índices que evalúan la RC de las empresas utilizan como variables dimensiones y atributos. Estas dos variables son las que se utilizan tradicionalmente en todos y cada uno de los índices de medición de RC, tanto para los índices empresariales como para los académicos. Para el caso del IRCEC, se ha utilizado la misma categorización, debido a que permite un mejor desarrollo de los elementos a evaluar. Esta doble perspectiva académico-empresarial permitió la realización de un benchmark (análisis comparativo) total de 49 dimensiones y 202 atributos (tabla 2). La revisión de estos índices fue condición necesaria para la creación de la propuesta de índice para medir la RC en toda la cadena de valor aplicable a las empresas de comunicación, con énfasis en la formulación esta cadena y la segmentación de grupos de interés. Con la creación de nuevas dimensiones más estrechamente relacionadas con las empresas de comunicación, finalmente el modelo cuenta con 5 dimensiones y 17 atributos.

El paso siguiente a la formulación de dimensiones y atributos significó el planteamiento de las afirmaciones y/o interrogantes que se expondrían a los stakeholders de una empresa concreta con el fin de evaluar la RC. Estos postulados se construyeron teniendo como premisa que fueran fáciles de entender y que dieran cuenta del atributo y de la dimensión correspondiente. A este filtraje se le aplicó una doble codificación de los autores para arrojar la propuesta final.

Tradicionalmente, para la evaluación de aspectos relacionados con la RC se utilizan como técnicas de medición la escala Likert, escala Guttman, escala Thurstone, escala de Stapel y el diferencial semántico de Osgood (Capriotti, 2009). Aunque las más utilizadas son el diferencial semántico y la escala Likert, se optó por la segunda debido a que permite medir actitudes mientras que, por el contrario, el diferencial semántico mide significados. Además, la escala Likert permite el análisis desde un enfoque cognoscitivo, afectivo y conductual (Cortada, 2004). 
Tabla 2. Comparativo de índices de RC.

\begin{tabular}{|c|c|c|c|c|}
\hline Autor Índice & Ámbito & Dimensiones & Atributos & Propuesta \\
\hline Aula \& Mantere (2008) & Académico & 4 & 14 & Enfoque en la reputación en línea de las empresas. \\
\hline Carrió (2013) & Académico & 4 & 28 & $\begin{array}{l}\text { Estudia los índices de referencia empresarial y propone } \\
\text { uno que aglutina a los cuatro analizados. }\end{array}$ \\
\hline Hannington (2006) & Académico & - & 16 & No propone un índice concreto, si preguntas de cómo evaluar la RC. \\
\hline Jackson (2004) & Académico & - & 21 & $\begin{array}{l}\text { Analiza la RC a partir de preguntas con tres } \\
\text { posibles respuestas: sí, no, no aplica. }\end{array}$ \\
\hline López \& Iglesias (2006) & Académico & 2 & 13 & $\begin{array}{l}\text { Basado en índices académicos. Enfoque en cultura } \\
\text { organizativa y reputación vinculada al negocio. }\end{array}$ \\
\hline $\begin{array}{l}\text { Mandelli \& } \\
\text { Cantoni (2010) }\end{array}$ & Académico & - & - & $\begin{array}{l}\text { No proponen índice. Proponen análisis de } \\
\text { contenido de información en web. }\end{array}$ \\
\hline $\begin{array}{l}\text { Martínez \& } \\
\text { Olmedo (2009) }\end{array}$ & Académico & 4 & 15 & $\begin{array}{l}\text { Basado en dos modalidades: reputación vinculada a } \\
\text { la empresa y reputación asociada al producto. }\end{array}$ \\
\hline Morley (2002) & Académico & 5 & 12 & $\begin{array}{l}\text { No es índice específico para reputación, pero evalúa } \\
\text { aspectos de imagen, RSC, entre otros. }\end{array}$ \\
\hline Schwaiger (2004) & Académico & 4 & - & Propuesta a partir de otros índices académicos, solo con atributos. \\
\hline $\begin{array}{l}\text { Schwaiger, Raithel \& } \\
\text { Schloderer (2009) }\end{array}$ & Académico & 2 & 6 & $\begin{array}{l}\text { Propuesta basada en seis preguntas dentro de dos } \\
\text { ámbitos de análisis: simpatía y competencia. }\end{array}$ \\
\hline $\begin{array}{l}\text { Shamma and } \\
\text { Hassan (2009) }\end{array}$ & Académico & - & 13 & $\begin{array}{l}\text { Basado en otros índices académicos. Se } \\
\text { enfoca en usuarios y no usuarios. }\end{array}$ \\
\hline Merco & Empresarial & 6 & 14 & $\begin{array}{l}\text { Rankings en España y Latinoamérica. Tiene } \\
\text { diferentes ámbitos de evaluación. }\end{array}$ \\
\hline Reptrak & Empresarial & 7 & 21 & Cuantifican la reputación de la empresa y proponen estrategias. \\
\hline RQ & Empresarial & 6 & 20 & $\begin{array}{l}\text { Utiliza proceso de nominación y evaluación. Utiliza } \\
\text { encuestas en internet a usuarios de las marcas. }\end{array}$ \\
\hline WMAC & Empresarial & 5 & 9 & $\begin{array}{l}\text { Rankings de reputación de empresas en el mundo. } \\
\text { Discriminación por países y por dimensiones. }\end{array}$ \\
\hline
\end{tabular}


Las escalas Likert tradicionalmente pueden contener 5, 7, 9 y 11 ítems; sin embargo, para Cortada (2004) las de cinco ítems cumplen con las mismas condiciones que una de escala mayor. Según esta condición, los cinco ítems utilizados fueron: totalmente en desacuerdo, en desacuerdo, indeciso, de acuerdo y totalmente de acuerdo. En total el IRCEC cuenta con 37 postulados que son formulados a través de preguntas cerradas mediante el uso de esta escala.

\section{Resultados}

La propuesta de un índice de RC para empresas de comunicación representa una labor de análisis de metodologías de evaluación de la RC, de exploración de empresas de comunicación, de segmentación de los stakeholders, entre otras actividades. El Índice de Reputación Corporativa para Empresas de Comunicación, IRCEC, posee 5 dimensiones y 17 atributos, en los que se han tenido en cuenta las condiciones más importantes de las empresas de comunicación en su relación con los stakeholders.

Para el caso del IRCEC, las dimensiones representan las magnitudes o tópicos generales del aspecto a evaluar, es decir, la medida orientativa que determina los atributos. También se pueden interpretar las dimensiones como la materialización de los aspectos preponderantes de la actuación de la organización con relación a sus stakeholders. Asimismo, los atributos serán las cualidades y propiedades específicas de cada una de las dimensiones, lo que equivale a decir que los atributos son representaciones concretas de cada dimensión (tabla 3).

Tabla 3. Índice de RC para empresas de comunicación.

\begin{tabular}{|c|c|c|}
\hline Dimensiones & Atributos & \\
\hline Autonomía & $\begin{array}{l}\text { Transparencia } \\
\text { Honestidad }\end{array}$ & $\begin{array}{l}\text { Independencia } \\
\text { Confianza }\end{array}$ \\
\hline Vínculo & $\begin{array}{l}\text { Diálogo } \\
\text { Empatía } \\
\text { Percepción }\end{array}$ & \\
\hline Calidad & $\begin{array}{l}\text { Calidad percibida } \\
\text { Garantía y atención } \\
\text { Acceso a información }\end{array}$ & \\
\hline RSC & $\begin{array}{l}\text { Responsabilidad social } \\
\text { Responsabilidad económica }\end{array}$ & $\begin{array}{l}\text { Responsabilidad ambiental } \\
\text { Responsabilidad legal }\end{array}$ \\
\hline Gestión & $\begin{array}{l}\text { Gestión estratégica } \\
\text { Estructura empresarial } \\
\text { Cultura organizacional }\end{array}$ & \\
\hline
\end{tabular}




\section{Dimensión autonomía}

La dimensión autonomía dentro del IRCEC se definirá bajo la perspectiva y concepción griega que la cataloga como la capacidad de gobernarse a sí mismo, es decir, el propio autogobierno en función del sistema de valores que posea la organización. Los atributos que definen y caracterizan esta dimensión son transparencia, honestidad, independencia y confianza. Con el objetivo de aplicar el IRCEC, se han relacionado ocho preguntas que permitirán conocer las percepciones de los stakeholders respecto de la autonomía de las empresas de comunicación (tabla 4).

Tabla 4. Preguntas correspondientes a la autonomía.

\begin{tabular}{ll}
\hline Autonomía & \\
\hline La empresa: & \\
\hline Transparencia & $\begin{array}{l}\text { - Informa a sus stakeholders de todas sus acciones } \\
\text { - Da a conocer a sus stakeholders informes con sus cuentas anuales }\end{array}$ \\
\hline Honestidad & - Demuestra ser una organización con claridad informativa \\
\hline Independencia & - No emite información falsa \\
\hline Confianza & - Es una organización neutral e imparcial \\
\hline
\end{tabular}

\section{Dimensión vínculo}

Se interpreta el vínculo dentro del desarrollo del índice como la interacción entre dos o más personas u organizaciones, de manera que se sostiene una conexión dinámica a través de una conducta específica que está determinada por los intereses de cada una de las partes. Los atributos que forman parte del vínculo son diálogo, empatía y percepción. Para cada uno de estos tres atributos se proponen dos preguntas (tabla 5).

Tabla 5. Preguntas correspondientes al vínculo.

\begin{tabular}{|c|c|}
\hline Vínculo & \\
\hline \multicolumn{2}{|l|}{ La empresa: } \\
\hline \multirow{2}{*}{ Diálogo } & Dispone de canales de comunicación bilateral \\
\hline & Es una organización que acepta opiniones contrarias \\
\hline \multirow{2}{*}{ Empatía } & Es una buena compañía para trabajar \\
\hline & Es una organización que demuestra familiaridad \\
\hline \multirow{2}{*}{ Percepción } & Es una organización que transmite respeto \\
\hline & Es una organización con una buena imagen \\
\hline
\end{tabular}




\section{Dimensión calidad}

La calidad se entenderá como las características cualitativas de una organización que le confieren la aptitud de satisfacer de la mejor manera posible las expectativas y necesidades de los stakeholders con los que tiene un vínculo. Los atributos que hacen parte de la dimensión calidad son calidad percibida, garantía y atención y acceso a información. Para cada uno de los atributos se han construido dos preguntas que permitirán la valoración completa de la calidad de la empresa de comunicación (tabla 6).

Tabla 6. Preguntas correspondientes a la calidad.

\begin{tabular}{ll}
\hline Calidad & \\
\hline La empresa: & $\begin{array}{l}\text { Ofrece a sus stakeholders productos que cumplen sus expectativas } \\
\text { Desarrolla productos innovadores }\end{array}$ \\
\hline Calidad percibida & $\begin{array}{l}\text { Dispone de productos para diversos stakeholders } \\
\text { Responde a las exigencias de los stakeholders }\end{array}$ \\
\hline Garantía y atención & $\begin{array}{l}\text { Ofrece facilidades de acceso a la información a sus stakeholders } \\
\text { Comunica a los stakeholders los canales disponibles para el diálogo }\end{array}$ \\
\hline
\end{tabular}

\section{Dimensión RSC}

La Responsabilidad Social Corporativa se representa dentro del análisis del IRCEC como el cumplimiento de las obligaciones de la organización con cada uno de sus stakeholders en los ámbitos económicos, sociales, medioambientales y legales. Ya que esta es una de las dimensiones más importantes del IRCEC para los efectos de la investigación, se han creado diez preguntas que dan cuenta de los cuatro atributos mencionados (tabla 7).

Tabla 7. Preguntas correspondientes a la RSC.

\begin{tabular}{ll}
\hline RSC & \\
\hline La empresa: & $\begin{array}{l}\text { Respeta los derechos humanos } \\
\text { Apoya las buenas causas } \\
\text { Promueve condiciones de equidad con sus stakeholders }\end{array}$ \\
\hline Responsabilidad social & $\begin{array}{l}\text { Combate la corrupción } \\
\text { No lleva a cabo prácticas de explotación laboral } \\
\text { Responsabilidad económica }\end{array}$ \\
\hline Responsabilidad ambiental & $\begin{array}{l}\text { Es una organización respetuosa con el medio ambiente } \\
\text { Desarrolla campañas para la conservación ambiental }\end{array}$ \\
\hline Responsabilidad legal & $\begin{array}{l}\text { Cumple las leyes } \\
\text { Dispone de códigos de conducta que aplica en toda la organización }\end{array}$ \\
\hline
\end{tabular}




\section{Dimensión gestión}

La gestión está representada por las acciones que desarrolla la organización con el objetivo de brindar un producto o servicio de calidad y que, además, mejore las condiciones de competitividad y productividad. Para esta dimensión se relacionan siete preguntas correspondientes a tres atributos: gestión estratégica, estructura empresarial y cultura organizacional (tabla 8 ).

Tabla 8. Preguntas correspondientes a la gestión.

\begin{tabular}{ll}
\hline Gestión & \\
\hline La empresa: & $\begin{array}{l}\text { Desarrolla su labor basada en procesos de planeación estratégica } \\
\text { Es una organización con una gestión financiera sólida }\end{array}$ \\
\hline Gestión estratégica & Es líder dentro de su sector \\
\hline Estructura empresarial & Posee una estructura empresarial que favorece su desarrollo \\
\hline Cultura organizacional & Ce adapta con facilidad a los cambios \\
\hline
\end{tabular}

\section{Modelo IRCEC}

Las preguntas cerradas de orden cuantitativo que se han construido a partir de los atributos son valoradas por los stakeholders a través de una escala Likert de cinco puntos. Ya que por cada dimensión se dispone de entre seis y diez preguntas, estas se han ubicado dentro del índice de manera aleatoria. La idea de mezclar las preguntas dentro del IRCEC pretende que no se creen secciones de contenido evidentes para quienes responden la encuesta y así aminorar los potenciales sesgos en las respuestas.

En definitiva, el IRCEC consta de 37 preguntas cerradas, en las que, para efecto de la encuesta a cada uno de los grupos de interés, solo varía la denominación según el tipo de stakeholder. Es decir, en la pregunta "La empresa promueve la participación de los empleados en las decisiones empresariales" se cambiará el enunciado según cada uno de los siete grupos de interés. Por ejemplo, para el caso de los proveedores la pregunta sería: "La empresa promueve la participación de los proveedores en las decisiones empresariales" (tabla 9). 
Tabla 9. Preguntas cuantitativas IRCEC.

\begin{tabular}{|c|c|c|c|c|c|}
\hline & $\begin{array}{l}\text { Totalmente } \\
\text { en desacuerdo }\end{array}$ & $\begin{array}{l}\text { En } \\
\text { desacuerdo }\end{array}$ & Indeciso & $\begin{array}{l}\text { De } \\
\text { acuerdo }\end{array}$ & $\begin{array}{l}\text { Totalmente } \\
\text { de acuerdo }\end{array}$ \\
\hline La empresa: & & & & & \\
\hline $\begin{array}{l}\text { - Desarrolla productos innovadores } \\
\text { - Es líder dentro de su sector } \\
\text { - Es una organización neutral e imparcial } \\
\text { - Es una organización que transmite respeto } \\
\text { - Contribuye al desarrollo del país } \\
\text { - Se adapta con facilidad a los cambios } \\
\text { - Informa a los proveedores de todas sus acciones } \\
\text { - Es una organización con una buena imagen } \\
\text { - Respeta los derechos humanos } \\
\text { - Combate la corrupción } \\
\text { - Dispone de productos para diversos públicos } \\
\text { - Es una organización que respeta a los proveedores cumple las leyes } \\
\text { - Posee una estructura empresarial que favorece su desarrollo } \\
\text { - Desarrolla campañas para la conservación ambiental } \\
\text { - Da a conocer a los proveedores informes con sus cuentas anuales } \\
\text { - Es una buena compañía para trabajar } \\
\text { - Dispone de códigos de conducta que aplica en toda la organización } \\
\text { - Cuenta con una apropiada cultura organizacional } \\
\text { - No lleva a cabo prácticas de explotación laboral } \\
\text { - No recibe influencias de empresas o de organizaciones externas } \\
\text { - Promueve la participación de los proveedores en las decisiones empresariales } \\
\text { - Es una organización que demuestra familiaridad } \\
\text { - Promueve condiciones de equidad con los proveedores } \\
\text { - Comunica a los proveedores los canales disponibles para el diálogo } \\
\text { - Desarrolla su labor basada en procesos de planeación estratégica } \\
\text { - Responde a las exigencias de los proveedores } \\
\text { - No emite información falsa } \\
\text { - Apoya las buenas causas } \\
\text { - Ofrece facilidades de acceso a la información a los proveedores } \\
\text { - Es una organización en la que se puede creer } \\
\text { - Dispone de canales de comunicación bilateral } \\
\text { - Es una organización respetuosa con el medio ambiente } \\
\text { - Demuestra ser una organización con claridad informativa } \\
\text { - Es una organización con una gestión financiera sólida } \\
\text { - Ofrece a los proveedores productos que cumplen sus expectativas } \\
\text { - Es una organización que acepta opiniones contrarias }\end{array}$ & & & & & \\
\hline
\end{tabular}

Finalmente, ya que el IRCEC pretende sinceridad y claridad en las respuestas, no se requiere información específica de los grupos de interés, lo que asegura su anonimato $^{12}$. Sin embargo, se solicita la información demográfica básica para conocer los aspectos más esenciales de los stakeholders: sexo, edad y perfil profesional. En definitiva, el IRCEC es una herramienta que permite evaluar la RC de las empresas de comunicación y se convierte en la base para entender las percepciones de todos los integrantes

\footnotetext{
12 También debe mantenerse el anonimato para cumplir los estándares éticos de la investigación que implica a
} humanos. 
de la cadena de valor. La aplicación de las preguntas cuantitativas y la posibilidad de modificar las preguntas cualitativas lo convierten en una opción importante para entender la forma en que la RSC o las acciones empresariales inciden en la RC.

\section{Discusión y conclusiones}

Como limitación de la investigación deber recordarse que esta es una propuesta conceptual y metodológica que no atiende a los resultados de la aplicación del IRCEC, sino a su construcción. La elaboración del estado de la cuestión, del análisis de empresas de comunicación y de los índices de RC existentes en el mundo empresarial y académico permite concluir que, en la actualidad, este índice especializado que evalúa la RC de las empresas de comunicación es novedoso desde distintos puntos de vista.

En primer lugar, la totalidad de índices académicos y empresariales estudiados enfocan la aplicación de sus herramientas a todo tipo de empresas, de tal forma que no hacen una segmentación de variables de acuerdo con diferentes sectores económicos. No existe un modelo completamente válido, no obstante, la creación del IRCEC integra modelos y técnicas de índices anteriores, a partir de una técnica de benchmarking y el análisis de diferentes propuestas de segmentación de los grupos de interés. La creación de un índice de RC para empresas de comunicación responde a esta necesidad de contar con herramientas de evaluación sectorial.

En segundo lugar, ni los índices de referencia académica ni los empresariales proponen formas de segmentación o delimitación de los stakeholders para definir la cadena de valor y que las mediciones se realicen teniendo en cuenta a toda la cadena de valor. Esta es una consideración necesaria para la aplicación de un modelo de evaluación de la RC si se pretende impactar a todos los grupos de interés. La delimitación de la cadena de valor y el conocimiento de sus necesidades mínimas es una condición vital para establecer un diálogo con los stakeholders.

Si bien las percepciones de un grupo pueden dar indicios de las demás partes interesadas, no se pueden extrapolar los resultados pues una marca o una empresa puede tener tantas reputaciones como grupos de interés. Así, la evaluación segmentada por stakeholders se propone como la vía más recomendable para posteriormente comparar percepciones y emprender estrategias diferenciadas para cada grupo de interés.

El IRCEC es un avance interesante en la evaluación de la RC en empresas de comunicación pues se entrega una herramienta especializada, que además de medir la Reputación Corporativa permite analizar las percepciones de los grupos de interés de toda la cadena de valor. Incluso, es el primer paso para la materialización de nuevos índices de RC específicos para uso especializado en otros sectores empresariales.

Las principales conclusiones de esta investigación están vinculadas al desarrollo de los métodos de evaluación de la RC en empresas de comunicación. Sin embargo, 
se pueden evidenciar aspectos relacionados con los stakeholders y la misma RC de las empresas de comunicación. La comprensión por parte de los stakeholders de las actuaciones de la empresa implica que una misma marca o empresa podrá tener diferentes reputaciones. Cada una de las partes interesadas puede tener una valoración diferenciada de la RC. Esta es una conclusión importante, pues allí radica una de las razones por las que la RC es un intangible difícil de controlar y a su vez difícil de cuantificar.

Una conclusión relacionada con los índices es que la gran mayoría de las propuestas utilizan como base para su implementación tanto dimensiones (magnitudes de los aspectos a evaluar) como atributos (representación de cada dimensión mediante cualidades y propiedades específicas). A través de esta metodología se generan categorías y subcategorías de análisis, lo que permite el establecimiento adecuado de las variables.

Igualmente, una estrategia encaminada a mejorar la RC de una empresa deberá identificar los valores intangibles de la marca que son importantes para sus grupos de interés, potenciarlos en la comunicación de la identidad para que posteriormente sean percibidos por los stakeholders. Aunque las bases sobre las que se construye la RC son múltiples, directivos y empresarios deberán reconocer cuáles son aquellos intangibles que más valoran sus grupos de interés, tanto intangibles derivados de la gestión empresarial (calidad, servicio, liderazgo), como intangibles emanados del comportamiento corporativo (honestidad, confianza, transparencia, RSC).

La identificación de los valores intangibles y de la concepción que tienen los grupos de interés sobre la RC de las empresas es uno de los aspectos más apremiantes en el desarrollo teórico y práctico de la Reputación Corporativa. Aunque los índices que evalúan la RC no pueden ser considerados como herramientas definitivas o infalibles debido a la dificultad de evaluar intangibles, el desarrollo de métodos de evaluación de la RC es una tarea que permite conocer mejor las expectativas de los stakeholders y los intangibles que más repercuten en la RC.

Esta es una propuesta que puede abrir un debate académico sobre la conveniencia o no de tener índices segmentados por sector de negocio. También sobre la adecuación de evaluar a toda la cadena de valor. Por ejemplo, el RQ realiza su evaluación únicamente indagando por la $\mathrm{RC}$ en el público general. Al respecto, hay investigadores que consideran que los usuarios de una marca no son los más adecuados para este tipo de evaluaciones pues no tienen el conocimiento suficiente para determinar la RC de una empresa (Carrió, 2011). Aunque los usuarios de una marca no conozcan todos los aspectos de la empresa no quiere decir que no tengan una concepción de su RC. Además, las TIC y las redes sociales han permitido que todos los grupos de interés accedan a información detallada de cualquier empresa (Orozco-Toro \& Ferré-Pavia, 2012).

El IRCEC se convierte en una herramienta que permite medir la RC en empresas de comunicación, tomando como base factores decisivos y específicos para este tipo de 
empresas. El estudio de las dimensiones y atributos, tanto de propuestas académicas como empresariales, genera un instrumento que no solo pretende determinar la RC de las empresas de comunicación, sino que además da una mayor relevancia a variables como la RSC y puede aplicarse en toda la cadena de valor. El procedimiento de análisis y filtrado de dimensiones y atributos, además de la creación de nuevas dimensiones acordes con las necesidades de las empresas de comunicación, dieron origen al IRCEC. Esta metodología de medición de la RC admite que se realicen cambios tanto en dimensiones y atributos según las prioridades de la empresa o las necesidades de los investigadores.

\section{Referencias}

Aguilera, J. \& Baños, M. (2016). Branded entertainment. Madrid: ESIC.

Alloza, A. (2006). La medición continua de la reputación corporativa. En Benavides, J., Fernández, J. and Villagra, N. (Eds.), La ética de la responsabilidad social de las empresas y organizaciones (pp. 183-204). Madrid: Universidad Pontificia Comillas. Alloza, A. (2015). Aproximación a la reputación y a la responsabilidad corporativa. En

Benavides, J. and Monfort, A. (Eds.), Comunicación y empresa responsable (pp. 171197). Pamplona: EUNSA.

Álvarez, J. T. (2008). Entorno mixto para gestión de reputación e intangibles. En Fernández, M. (Ed.), Comunicación en la sociedad red: la construcción mediática de la realidad (pp. 73-95). Ávila: Universidad Católica de Ávila.

Argenti, P. \& Forman, J. (2002). The power of corporate communication. Nueva York: McGraw Hill.

Argenti, P. (2014). Comunicación estratégica y su contribución a la reputación. Madrid: LID.

Arrese, A. \& Baigorri, M. (2011). Corporate reputation and the news media in Spain.

En Carroll, C. (Ed.), Corporate reputation and the news media (pp. 168-191). New York: Routledge.

Aula, P. \& Mantere, S. (2008). Strategic reputation management. Nueva York: Routledge. Capriotti, P. (2009). Branding corporativo. Fundamentos para la gestión estratégica de la identidad corporativa. Santiago: Andros.

Carrió, M. (2011). Creació d’una nova metodologia multistakeholder per a l'amidament de la reputació corporativa a partir de lanàlisi de les 5 metodologies de referència: Fortune AMAC, Fortune WMAC, Merco, Coeficient de Reputació Corporativa (CRQ) i RepTrack (Tesis de doctorado). Universidad Pompeu Fabra, Barcelona. Recuperado de http://www.upf.edu/doctorats/en/menutesi/tesis/11.html.

Carrió, M. (2013). Gestión de la reputación corporativa. Barcelona: Libros de Cabecera. Caruana, A. (2008). An attitudinal measure of corporate reputation. En Melewar, T. 
(Ed.), Facets of corporate identity, communication and reputation (pp. 197-209). Nueva York: Routledge.

Cortada, N. (2004). Teoría y métodos para la construcción de escalas de actitudes. Buenos Aires: Lugar.

Costa, J. (2009). El DirCom hoy. Dirección y gestión de la comunicación en la nueva economía. Barcelona: Costa Punto Com.

Davies, G., Chun, R., Vinhas, R. \& Roper, S. (2003). Corporate reputation and competitiveness. Nueva York: Routledge.

Delgado, V. \& Olarte, M. (2012). Responsabilidad social corporativa en el sector de la televisión. Un estudio longitudinal de las memorias de sostenibilidad. Revista Internacional de Investigación en Comunicación AdResearch ESIC, 6(6), 112-129.

Doorley, J. \& García, H. (Eds.) (2011). Reputation management. The key to successful public relations and corporate communication. Nueva York: Routledge.

Dowling, G. (2001). Creating corporate reputations. Identity, image, and performance. Nueva York: Oxford University Press.

Earl, S. \& Waddington, S. (2012). Brand anarchy. Managing corporate reputation. Londres: Bloomsbury.

Etkin, E. (2008). El recorrido metodológico de la auditoría. En Suárez, A. (Ed.), Auditoría de comunicación (pp. 61-96). Buenos Aires: La Crujía.

Fernández, M. (2011). Comunicación y reputación en empresas e instituciones. Experiencias profesionales y propuestas prácticas. Madrid: Universitas.

Fernández, T. (2013).Las corporaciones de radiotelevisión pública de la Unión Europea y el incipiente uso de la web 2.0 para comunicar RSC. Journal of Communication, 6, 86-110.

Firestein, P. (2009). Crisis of character. Building corporate reputation in the age of skepticism. Nueva York: Union Square Press.

Fombrun, C. (1996). Reputation. Realizing value from the corporate imagen. Boston: Harvard Business School Press.

Fombrun, C. \& Rindova, V. (2000). The road to transparency: reputation management at Royal Dutch/Shell. En Schultz, M., Hatch, M. and Larsen, M. (Eds.), The expressive organization. Linking identity, reputation, and the corporate brand (pp. 77-96). Nueva York: Oxford University Press.

Freeman, B. (2006). Substance sells: aligning corporate reputation and corporate responsibility. Public Relations Quaterly, 51(1), 12-19.

Gable, T. (2008). Imagen as a part of corporate strategy. Building reputation for long-term benefit. En Anthonissen, P. (Ed.), Crisis Communication. Practical PR strategies for reputation management and company survival (pp.44-54). Londres: Kogan Page.

Gaultier, S., Louisot, J. \& Rayner, J. (2009). Managing reputational risk - from theory to 
practice. En Klewes, J. and Wreschniok, R. (Eds.), Reputation capital. Building and maintaining trust in the 21st century (pp. 161-176). Londres: Springer.

Griffin, A. (2009). New strategies for reputation management. Gaining control of issues, crises and corporate social responsibility. Londres: Kogan Page.

Hannington, T. (2006). Cómo medir y gestionar la reputación de su empresa. Barcelona: Deusto.

Hernández, M., Losada, A. \& Macías, A. (2007). Estrategia y conducta social de la organización. Salamanca: Universidad Pontificia Salamanca.

Huang, L., Wang, S., Hsu, C., Zhang, J. \& Yang, F. (2016). Using reputation measurement to defend mobile social networks against malicious feedback ratings. The Journal of Supercomputing, 71(6), 2190-2203. doi: https://doi.org/10.1007/s1122.

Ingenhoff,D.\& Koelling,M.(2012).Mediagovernanceand corporate social responsibility of media organizations: an international comparison. Business Ethics: A European Review, 21(2), 154-167. doi: https://doi.org/10.1111/j.1467-8608.2011.01646.x.

Jackson, K. (2004). Building reputational capital. Strategies for integrity and fair play that improve the bottom line. Nueva York: Oxford University Press.

Komisarjevsky, C. (2012). The power of reputation. Strengthen the asset that will make or break your career. Nueva York: Amacom.

Larkin, J.(2003).Strategic reputation risk management. Nueva York: Palgrave MacMillan. Lindemann, J. (2010). El valor financiero de la marca. En Brujó, G. (Ed.), En clave de marcas (pp. 48-65). Madrid: LID.

Lizcano, J. (2006). Información empresarial sobre responsabilidad social. En Benavides, J., Fernández, J. \& Villagra, N. (Eds.), La ética y la responsabilidad social de las empresas y organizaciones (pp. 121-140). Madrid: Universidad Pontificia Comillas. López, V. \& Iglesias, S. (2006). Percepciones directivas del recurso reputación. Estudio empírico de sus relaciones con el rendimiento empresarial. Cuadernos de Economía y Dirección de la Empresa, 28, 139-160.

López, B. \& Sebastián, A. (2009). Responsabilidad social corporativa y reputación corporativa. En Sánchez, J. and Pintado, T. (Eds.), Imagen corporativa. Influencia en la gestión empresarial (pp. 139-170). Madrid: ESIC.

Mandelli, A. \& Cantoni, L. (2010). Social media impact on corporate reputation: proposing a new methodological approach. Cuadernos de Información, 27, 61-74. doi: https://doi.org/10.7764/cdi.27.23.

Martínez,I.\&Olmedo,I.(2009).La medición de la reputación empresarial:problemática y propuesta. Investigaciones Europeas, 15(2), 127-142.

Merino, M. \& Pintado, T. (2009). Auditoría y estrategia de imagen. En Sánchez, J. and Pintado, T. (Eds.), Imagen corporativa. Influencia en la gestión empresarial (pp. 83104). Madrid: ESIC. 
Morley, M. (2002). How to manage your global reputation. Nueva York: Palgrave.

Nomen, E. (2005). Valor razonable de los activos intangibles. El efecto mariposa de la segunda deslocalización. Barcelona: Deusto.

Olins, W. (2000). How brands are taking over the corporation. En Schultz, M., Hatch, M. \& Larsen, M. (Eds.), The expressive organization. Linking identity, reputation, and the corporate brand (pp. 51-65). Nueva York: Oxford University Press.

Orozco-Toro, J. \& Ferré-Pavia, C. (2012). La fuerza de los stakeholders en el caso de La Noria. Ciudadanía crítica y uso de las redes sociales en un análisis de la reputación corporativa. Icono 14, 10(3), 403-424. doi: https://doi.org/10.7195/ri14.v10i3.159.

Orozco-Toro, J. \& Ferré-Pavia, C. (2017). La percepción de la reputación corporativa de los stakeholders de una empresa de comunicación: cómo afecta a Televisió de Catalunya comunicar una acción de responsabilidad social corporativa. Palabra Clave, 20 (2), 473-505. doi: https://doi.org/10.5294/pacla.2017.20.2.8.

Paranaíba, I., Souza, R., Marques, A. \& Miranda, J. (2016). Efeitos da reputação corporativa no custo de capital próprio em empresas brasileiras listadas. Revista Universo Contábil, 12(1), 178-198. doi: https://doi.org/10.4270/ruc.2016110.

Pérez, A. \& Rodríguez del Bosque, I. (2014). Identidad, imagen y reputación de la empresa: integración de propuestas teóricas para una gestión exitosa. Cuadernos de Gestión, 14(1), 97-126. doi: https://doi.org/10.5295/cdg.130389ap.

Quevedo, E. (2003). Reputación y creación de valor. Madrid: Thomson.

Raithel, S., \& Schwaiger, M. (2015). The effects of corporate reputation perceptions of the general public on shareholder value. Strategic Management Journal, 36(6), 945-956.

Rodríguez, M. \& Alfaro, J. (2004). La sostenibilidad como factor de creación de valor para las empresas. En Fundación Empresa y Sociedad.(Eds.), Manual de la empresa responsable (pp. 9-19). Madrid: Cinco Días.

Ruiz, B., Esteban, A., \& Gutiérrez, S. (2014). Reputación bancaria durante la crisis económica. Comparación entre las principales entidades financieras desde la perspectiva del cliente. Universia Business Review, 43, 16-35.

Salinas, G. (2007). Valoración de marcas. Bilbao: Deusto.

Sanna, D. (2013). Desafíos actuales frente a la medición de la reputación corporativa. Austral Comunicación, 2(1), 79-104.

Sanz de la Tajada, L. (1994). Integración de la identidad y la imagen de la empresa. Madrid: ESIC.

Sarstedt, M., Wilczynski, P., \& Melewar, T. C. (2013). Measuring reputation in global markets-A comparison of reputation measures' convergent and criterion validities. Journal of World Business, 48(3), 329-339. doi: https://doi.org/10.1016/j. jwb.2012.07.017. 
Schultz, M., Hatch, M. \& Larsen, M. (Eds.) (2000). The expressive organization. Linking identity, reputation, and the corporate brand. Nueva York: Oxford University Press.

Schwaiger, M. (2004). Components and parameters of corporate reputation - an empirical study. Schmalenbach Business Review, 56, 46-71.

Schwaiger, M., Raithel, S. \& Schloderer, M. (2009). Recognition or rejection - How a company's reputation influences stakeholder behaviour. En Klewes, J. and Wreschniok, R. (Eds.), Reputation Capital. Building and maintaining trust in the 21st century (39-52). Londres: Springer.

Shamma, H. \& Hassan, S. (2009). Customer and non-customer perspectives for examining corporate reputation. Journal of Product and Brand Management, 18(5), 326-337. doi: https://doi.org/10.1108/10610420910981800.

Tejedo-Romero, F. \& Ferraz, J.F. (2015). Información del Capital Humano: la generación de intangibles y la responsabilidad social. Cuadernos de Gestión, 16(1), 125-144. doi: https://doi.org/10.5295/cdg.140498ft.

Valenzuela, L., Jara-Bertin, M. \& Villegas, F. (2015). Prácticas de responsabilidad social, reputación corporativa y desempeño financiero. Revista de Administração de Empresas, 55(3), 329-344. doi: https://doi.org/10.1590/S0034-759020150308.

Van Riel, C. (1997). Comunicación corporativa. Madrid: Prentice Hall.

Vila, N. \& Küster, I. (2013). Antecedentes de reputación corporativa en cadenas televisivas. Revista de Ciencias Sociales, 19(2), 334-348.

Villafañe, J. (2004). La buena reputación. Claves del valor intangible de las empresas. Madrid: Pirámide. 